\title{
DIRECT APPROACH TO SOLVE NONHOMOGENEOUS DIFFUSION PROBLEMS USING FUNDAMENTAL SOLUTIONS AND DUAL RECIPROCITY METHODS
}

\author{
Der-Liang Young*, Chia-Cheng Tsai, and Chia-Ming Fan
}

\begin{abstract}
This paper describes a combination of the method of fundamental solutions (MFS) and the dual reciprocity method (DRM) as a mesh-free numerical method (MFS-DRM model) to solve 2D and 3D nonhomogeneous diffusion problems. Using our method, the homogeneous solutions of the diffusion equations are solved by the MFS, and the DRM, based on the radial basis functions (RBF) of the thin plate splines (TPS), is employed to solve for particular solutions. The present scheme is free from the frequently used Laplace transform and the finite difference discretization method to deal with the time derivative term in the governing equation. By properly placing the source points in the time-space domain, the solution is advanced in time until a steady state solution (if one exists) is reached. Since the present method does not need mesh discretization and nodal connectivity, the computational effort and memory storage required are minimal as compared to other domain-oriented numerical schemes such as FDM, FEM, FVM, etc. Test results obtained for 2D and 3D diffusion problems show good comparability with analytical solutions and other numerical solutions, such as those obtained by the MFS-DRM model based on the modified Helmholtz fundamental solutions. Thus the present numerical scheme has provided a promising meshfree numerical tool to solve nonhomogeneous diffusion problems with space-time unification for diffusion fundamental solutions.
\end{abstract}

Key Words: nonhomogeneous diffusion equation, method of fundamental solutions, dual reciprocity method, diffusion fundamental solution, multidimensions.

\section{INTRODUCTION}

There are many physical phenomena governed by diffusion equations, such as flow problems, heat transfer, solute transports, chemical processes and among others. Though analytical solutions can be obtained for some cases, the complexity in geometry generally necessitates the use of numerical methods. Classical numerical methods such as the finite difference, finite element, finite volume, and boundary element methods have been

*Corresponding author. (Tel: 886-2-23626114; Email: dlyoung@hy.ntu.edu.tw)

D. L. Young and C. M. Fan are with the Department of Civil Engineering, National Taiwan University, Taipei, Taiwan 106, R.O.C.

C. C. Tsai is with the Hydrotech Research Institute, National Taiwan University, Taipei, Taiwan 106, R.O.C. extensively adopted to solve various types of diffusion problems. Chawla and Al-Zanaidi (1999) and Hobson et al. (1996) applied the finite difference method to solve diffusion problems, and Teixeira (1999) carried out the analysis of the numerical stability of the problem. Moreover, Oden et al. (1998) employed a finite element method to solve the diffusion process in an unbounded medium. On the other hand, Jones and Menzies (2000) used the cell-centered finite volume method for the diffusion equation. Also, Zhu (1998), Zhu et al. (1998), Bulgakov et al. (1998), Zerroukat (1999), Sutradhar et al. (2002), and Bialecki et al. (2002) applied the BEM to solve diffusion equations. These classical methods are easy to apply for regular geometries. However, when the geometry is not regular, the mesh discretization requires a significant computational effort and a large amount of memory storage, especially for 3D problems, 
which is a big drawback for conventional domain-oriented methods.

In the past years, there has been an increasing interest in the idea of mesh-free numerical methods for solving partial differential equations (PDEs). Generally speaking, such methods can be divided into three categories. The first category is the so-called MFS-DRM method, which combines the method of fundamental solutions and the dual reciprocity method. The second type is the so-called Kansa's method (or multiquadrics (MQ) method) (Kansa, 1990a; 1990b), and the third type are so-called meshless local Petrov-Galerkin (MLPG) and local boundary integral equation (LBIE) methods based on integral equations (Atluri and Zhu, 1998; Zhu et al., 1998; Wordelman et al., 2000; Lin and Atluri, 2000; Kim and Atluri, 2000; Lin and Atluri, 2001). In this paper, we focus on the MFS-DRM model based on nonhomogeneous diffusion equations by the spacetime collocation technique.

In applications of the traditional boundary element method, a lot of computational effort is required to calculate the domain integration for the source term. The DRM was thus first introduced by Nardini and Brebbia (1982) to transform the domain integral to a boundary type by a series of RBF in their pioneer work. On the other hand, the MFS is used to approximate the homogenous solution by a series of fundamental solutions. The boundary conditions of the problem are satisfied by the boundary collocation method, and then the solution in the whole domain can be obtained. The present MFS-DRM method is free from the singular integral evaluation as required by the boundary element method. Therefore, the MFS-DRM method as a mesh-free numerical algorithm, has been considered to solve many PDEs in different areas successfully (Golberg, 1995; Chen et al., 1998b; Poullikkas et al., 1998b; Berger and Karageorghis, 1999; Golberg and Chen, 1998; Balakrishnan and Ramachandran, 2000; Smyrlis et al., 2001; Ramachandran, 2002). Golberg (1995) used the MFS to obtain a numerical solution of a Poisson equation. Poullikkas et al. (1998a) solved harmonic and biharmonic boundary value problems by the MFS. Chen et al. (1998a) applied the combination of MFS and the quasi-Monte-Carlo method for diffusion equations. We utilize the MFS-DRM model to solve nonhomogeneous diffusion problems in this paper, which is an extension for the direct solution of the homogeneous diffusion equation (Young et al., 2004).

In the literature, the solution of diffusion equations utilizing the MFS either use the Laplace transform (Chen et al., 1998b) or the finite difference scheme (Golberg and Chen, 1998; Balakrishnan and Ramachandran, 2000) to deal with the time derivative. This is due to the fact that the MFS is well treated in the spatial domain after the treatment of the transient part. In Chen et al.'s (1998b) work, they transformed the diffusion equation to the modified Helmholtz equation using the Laplace transform and then used the modified Helmholtz fundamental solution to solve the problems. When the Laplace transform is adopted, the inverse transform will be needed and sometimes it leads to certain difficulties in the solution process. Moreover, the particular solutions of the modified Helmholtz operator are mathematically more difficult than the particular solutions of the diffusion operator, in which only inverse the Laplace is required, if the source term is assumed time-independent. The same drawback as encountered in the Laplace transform approach is encountered when the time derivative is discretized by the finite difference scheme, since it finally also results in the modified Helmholtz equation with source term.

In this paper, the fundamental solution of the diffusion equation is directly used to obtain the homogeneous solution of the problem without the need for the Laplace transform or finite difference method as presented of early works. On the other hand, the DRM is utilized to solve the particular solution stemming from the nonhomogeneous source term, which is assumed to be quasi-static (Chen et al., 1996). This assumption is suitable since the source term is nearly a temporal distribution when using a small enough time step. Of course, further research is worthwhile for general cases of time-dependent sources. Since any diffusion problem is a time evolution process, the diffusion process can be obtained in a number of time steps by using the combination of the MFS and DRM methods. The proposed method is adopted to find numerical solutions in 2D and 3D geometries. As a first attempt the method is applied for a set of problems with the Dirichlet boundary conditions. Moreover, numerical solutions, which are obtained from the MFS-DRM model, based on the modified Helmholtz fundamental solution, as well as analytical solutions, are obtained for comparison purposes.

\section{GOVERNING EQUATION}

Consider a linear diffusion equation with timeindependent nonhomogeneous sources over a computational domain $\Omega$ with boundary $\Gamma$,

$$
\frac{\partial \Phi(x, t)}{\partial t}=k \nabla^{2} \Phi(x, t)+A(x)
$$

in which $\boldsymbol{x}$ is the general spatial coordinate, $t$ is the time, $k$ is the diffusion coefficient, $A(\boldsymbol{x})$ is the source function, and $\Phi(x, t)$ is the scalar variable to be determined. The initial condition of the diffusion problem is 


$$
\Phi(x, 0)=B(x) \text { in } \Omega
$$

with the Dirichlet and Neumann boundary conditions

$$
\begin{aligned}
& \Phi(\boldsymbol{x}, t)=C(\boldsymbol{x}, t) \text { on } \Gamma^{1} \\
& \frac{\partial \Phi}{\partial n}(\boldsymbol{x}, t)=D(\boldsymbol{x}, t) \text { on } \Gamma^{2}
\end{aligned}
$$

where $\Gamma^{1}+\Gamma^{2}$ is equal to the boundary $\Gamma$ and $n$ is the normal direction. Moreover, the boundary condition is of Dirichlet type if only $\Gamma^{2}=O$, of Neumann type if only $\Gamma^{1}=O$, and of Robin type if both $\Gamma^{1} \neq O$ and $\Gamma^{2} \neq O$. The augmented data of the problem are $A(\boldsymbol{x}), B(\boldsymbol{x})$, $C(\boldsymbol{x}, t), D(\boldsymbol{x}, t)$.

\section{NUMERICAL METHOD}

Generally speaking, there are two alternative MFS-DRM models which are capable of solving diffusion equations. They are the MFS-DRM model based on the diffusion fundamental solution, which is the main scheme to be derived here, and the MFSDRM model based on the modified Helmholtz fundamental solution to be revisited in the appendix (Chen et al., 1998b; Golberg and Chen, 1998).

First of all, we derive the fundamental solution of the linear diffusion equation, which is governed by

$$
\frac{\partial G(x, t ; \xi, \tau)}{\partial t}=k \nabla^{2} G(x, t ; \xi, \tau)+\delta(x, t ; \xi, \tau)
$$

By taking the Fourier transform with respect to $\boldsymbol{x}$ and the Laplace transform for $t$ and then inverting the transforms; the fundamental solution of the diffusion equation can be obtained as

$$
G(x, t ; \xi, \tau)=\frac{e^{-|x-\xi|^{2} / 4 k(t-\tau)}}{(4 \pi k(t-\tau))^{m / 2}} H(t-\tau)
$$

where $m$ is the spatial dimension number and $H(t)$ is the Heaviside step function.

Then, we formulate the MFS-DRM model by decomposing the solution into homogeneous and particular solutions as follows:

$$
\Phi(x, t)=\Phi_{h}(x, t)+\Phi_{p}(x)
$$

in which the particular solution, $\Phi_{p}(\boldsymbol{x})$, is a time-independent function which represents a steady state (or quasi-static) solution (Chen et al., 1996), and satisfies

$$
\nabla^{2} \Phi_{p}(\boldsymbol{x})+\frac{A(\boldsymbol{x})}{k}=0
$$

On the other hand, the time-dependent homogeneous solution, $\Phi_{h}(\boldsymbol{x}, t)$, which represents a transient (or dynamic) solution, satisfies the homogeneous diffusion equation as well as the modified initial and boundary conditions:

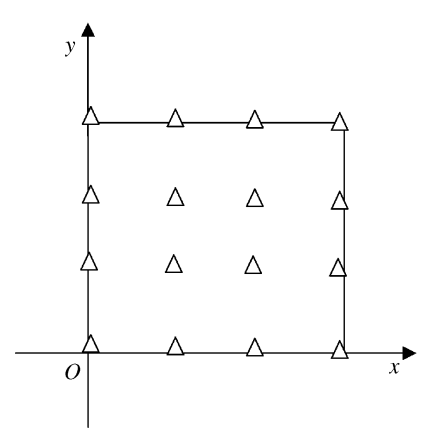

Fig. 1 Collocation points for the dual reciprocity method

$$
\begin{aligned}
& \frac{\partial \Phi_{h}(\boldsymbol{x}, t)}{\partial t}=k \nabla^{2} \Phi_{h}(\boldsymbol{x}, t) \text { in } \Omega \\
& \Phi_{h}(\boldsymbol{x}, 0)=B(\boldsymbol{x})-\Phi_{p}(\boldsymbol{x}) \text { in } \Omega \\
& \Phi_{h}(\boldsymbol{x}, t)=C(\boldsymbol{x}, t)-\Phi_{p}(\boldsymbol{x}) \text { on } \Gamma^{1} \\
& \frac{\partial \Phi_{h}}{\partial n}(\boldsymbol{x}, t)=D(\boldsymbol{x}, t)-\frac{\partial \Phi_{p}}{\partial n}(\boldsymbol{x}) \text { on } \Gamma^{2}
\end{aligned}
$$

The particular solution corresponding to Eq. (7) can be approximated by the DRM for the source term $-\frac{A(\boldsymbol{x})}{k}$.

$$
-\frac{A(\boldsymbol{x})}{k}= \begin{cases}\sum_{j=1}^{N} a_{j} r_{i j}^{2} \operatorname{Ln}\left[r_{i j}\right]+b_{1} x+b_{2} y+b_{3} & \text { for } 2 D \\ \sum_{j=1}^{N} a_{j} r_{i j}+b_{1} x+b_{2} y+b_{3} z+b_{4} & \text { for } 3 D\end{cases}
$$

where $r_{i j}=\left|\boldsymbol{x}_{i}-\boldsymbol{x}_{j}\right|$ is the radial distance between collocation points $\boldsymbol{x}_{j}$ and $\boldsymbol{x}_{i}$, and $N$ is the number of collocation points. Here, the collocation points are typically distributed in the interior domain as well as on the boundary (Fig. 1). After applying Eq. (9) in $N$ collocation points and the following augmented conditions, the unknown $a_{j}$ 's and the $b$ 's can be solved (Madych, 1992).

$$
\left\{\begin{array}{l}
\sum_{j=1}^{N} a_{j}=0 \\
\sum_{j=1}^{N} a_{j} x_{j}=0 \text { for } 2 D \\
\sum_{j=1}^{N} a_{j} y_{j}=0 \\
\sum_{j=1}^{N} a_{j}=0 \\
\sum_{j=1}^{N} a_{j} x_{j}=0 \\
\sum_{j=1}^{N} a_{j} y_{j}=0 \\
\sum_{j=1}^{N} a_{j} z_{j}=0
\end{array} \text { for } 3 D\right.
$$


Therefore, the particular solution $\Phi_{p}(x)$ can be determined (Golberg and Chen, 1998):

$$
\Phi_{p}(\boldsymbol{x})=\left\{\begin{array}{cc}
\sum_{j=1}^{N} a_{j} \frac{r_{i j}^{2}\left(-2 r_{i j}^{2}+4 r_{i j}^{2} \operatorname{Ln}\left[r_{i j}\right]\right)}{6144} \\
+b_{1} \frac{x^{3}}{6}+b_{2} \frac{y^{3}}{6}+b_{3} \frac{x^{2}+y^{2}}{4} \text { for } 2 D \\
\sum_{j=1}^{N} a_{j} \frac{r_{i j}^{3}}{12}+b_{1} \frac{x^{3}}{6}+b_{2} \frac{y^{3}}{6} \\
+b_{3} \frac{z^{3}}{6}+b_{4} \frac{x^{2}+y^{2}+z^{2}}{6} & \text { for } 3 D
\end{array}\right.
$$

With the substitution of the Eq. (1) into the modified initial and boundary conditions of the homogeneous Eq. (8), the result will be a well-posed homogeneous equation. The MFS is then applied to solve the equation. Since the diffusion fundamental solution satisfies the homogeneous diffusion equation, the solution can be assumed to be a linear combination of the fundamental solution of the diffusion operator. Therefore, the numerical solutions of the diffusion equation will be of the following form

$$
\Phi_{h}(x, t)=\sum_{j=1}^{N_{i}+N_{b}} c_{j} G\left(x, t ; \xi_{j}, \tau_{j}\right)
$$

where $x$ represents the location of the field points and $\xi_{j}$ gives the location of the source points. Moreover, $t$ and $\tau_{j}$ are the time of the field and source points respectively, $N_{i}$ and $N_{b}$ are the number of initial and boundary source points and $c_{j}$ 's are the undetermined coefficients, which can be determined by the method of collocation. The distributions of the field points and the source points are illustrated in Fig. 2 for a 2D situation. In Fig. 2, the field points are placed at the boundary portion at $t=(n+1) \Delta t$ and at the interior domain at $t=n \Delta t$. The source points are placed in the same position but at different time levels. By collocating these field points and using Eq. (8), a linear matrix system with dimension $N_{i}+N_{b}$ can be formed as follows:

$$
\left\lfloor A_{i j}\right\rfloor\left\{c_{j}\right\}=\left\{d_{i}\right\}
$$

$$
\text { where } A_{i j}= \begin{cases}\frac{e^{-\left|\boldsymbol{x}_{i}-\xi_{j}\right|^{2} / 4 k\left(t_{i}-\tau_{j}\right)}}{\left(4 \pi k\left|t_{i}-\tau_{j}\right|\right)^{m / 2}} & \text { for } t_{i}>\tau_{j} \\ 0 & \text { for } t_{i} \leq \tau_{j}\end{cases}
$$

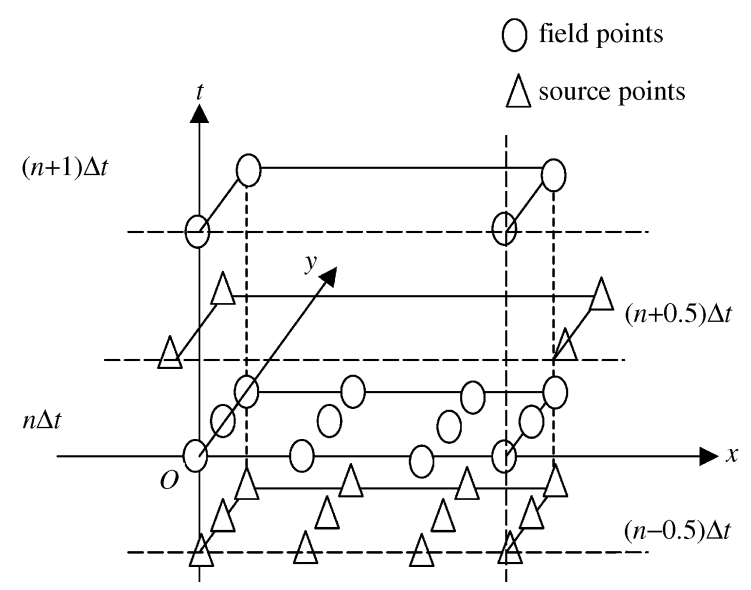

Fig. 2 Schematic diagram of source and field points for the MFS based on diffusion fundamental solution

The vector $\left\{d_{i}\right\}$ is the combination of initial and boundary conditions. After inverting the matrix system, the coefficients $\left\{c_{j}\right\}$ can be obtained, and then the homogenous solution at $t=(n+1) \Delta t$ can be acquired from Eq. (12).

If the homogeneous solution $\Phi_{h}(x, t)$ and the particular solution $\Phi_{p}(\boldsymbol{x})$ are solved, the solution $\Phi(\boldsymbol{x}, t)$ of the original diffusion equation is obtained by using the superposition principle of Eq. (6). Therefore, the procedure can be repeated until either the terminal time or a steady state solution is achieved.

\section{RESULTS AND DISCUSSIONS}

The proposed numerical scheme, the MFS-DRM model based on the diffusion fundamental solution, is validated by comparing the results obtained with the analytical solutions for diffusion problems with Dirichlet boundary conditions. Moreover, comparisons with the MFS-DRM model based on the modified Helmholtz fundamental solution are also carried out. The effectiveness of the method is verified by solving 2D and 3D nonhomogeneous diffusion problems and the results obtained are discussed in the following sections. The collocation points of the $2 \mathrm{D}$ DRM for the particular solutions are shown in Fig. 1. On the other hand, the source points and the field points of the MFS based on the diffusion fundamental solution are depicted in Fig. 2 for a 2D problem, and the points for the 2D MFS based on the modified Helmholtz fundamental solution are described in Fig. 3.

\section{2D Diffusion Problem}

The proposed method is utilized to study two examples of 2D diffusion problems in a square slab of size $[0,1] \times[0,1]$. 


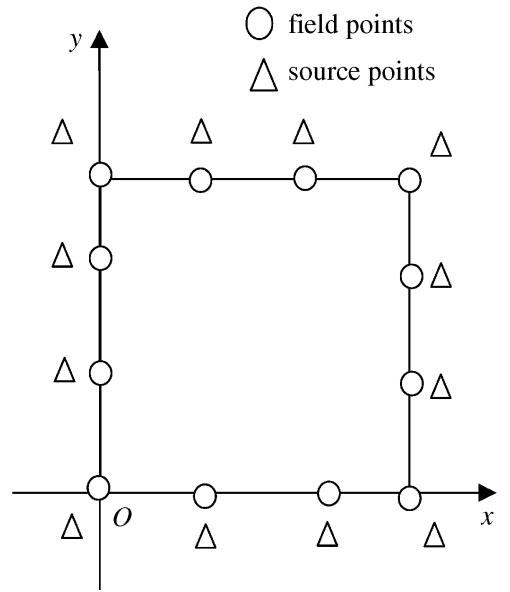

Fig. 3 Schematic diagram of source and field points for the MFS based on modified Helmholtz fundamental solution

\section{Example 1:}

G.E.: $\frac{\partial u}{\partial t}=\nabla^{2} u-\frac{6 x+6 y+2}{12}$

I.C.: $u(x, y, t=0)$

$=2(\cos [\pi x]+\sin [\pi y])+\frac{x^{3}+y^{3}+x^{2}+y}{12}$

B.C.: $\left\{\begin{array}{l}u(0, y, t)=2(1+\sin [\pi y]) e^{-\pi^{2} t}+\frac{y^{3}+y}{12} \\ u(1, y, t)=2(-1+\sin [\pi y]) e^{-\pi^{2} t}+\frac{y^{3}+y+2}{12} \\ u(x, 0, t)=2 \cos [\pi x] e^{-\pi^{2} t}+\frac{x^{3}+x^{2}}{12} \\ u(x, 1, t)=2 \cos [\pi x] e^{-\pi^{2} t}+\frac{x^{3}+x^{2}+2}{12}\end{array}\right.$

The analytical solution of the problem is given by

$u(x, y, t)$

$=2(\cos [\pi x]+\sin [\pi y]) e^{-\pi^{2} t}+\frac{x^{3}+y^{3}+x^{2}+y}{12}$

The comparison between the MFS-DRM model, based on the diffusion fundamental solution, and the analytical solution of the $u$-distribution along $y=0.5$ is depicted in Fig. 4. The results show generally good agreement, with the analytical solution in different time stages. In the figure, the gradient between the end surfaces decreases as time increases, thus approaching a steady state condition. The variations clearly demonstrate the physics underlying the diffusion process. Moreover, Fig. 5 and Fig. 6 show the time evolution history at $(0.5,0.5)$ of the solution and the absolute error, respectively, for the MFS-DRM model based on the diffusion fundamental solution, and for the MFS-DRM model based on the modified

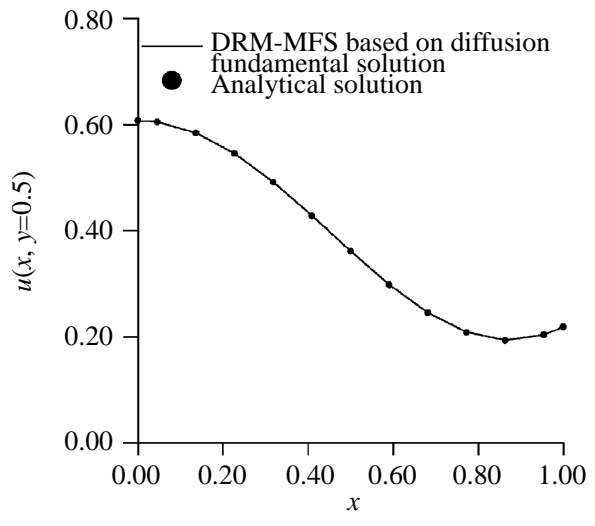

(a) $t=0.2$

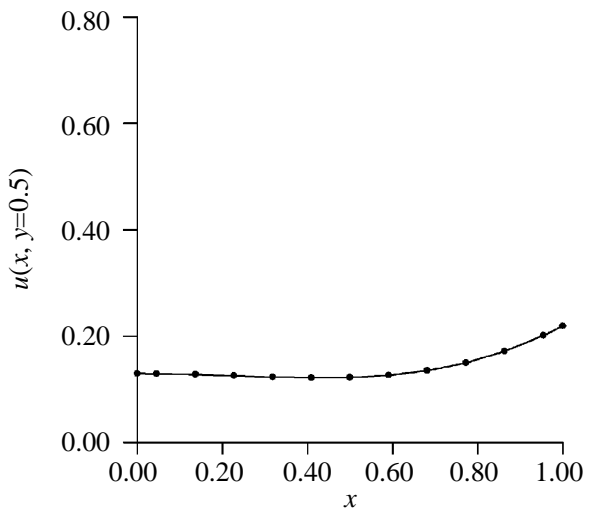

(b) $t=0.4$

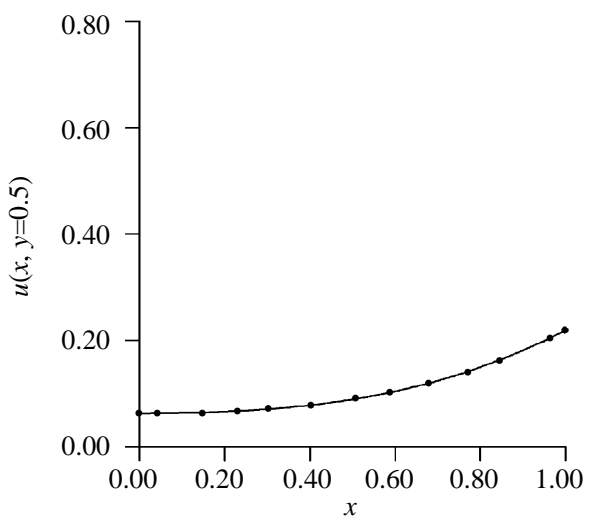

(c) $t=0.6$

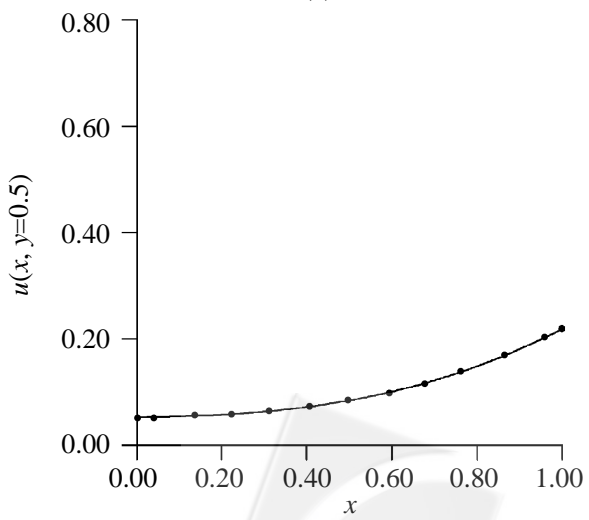

(d) $t=0.8$

Fig. 4 Comparison of $u$-distribution along $y=0.5$ by the MFS-DRM model based on diffusion fundamental solution (a) $t=0.2$; (b) $t=0.4$; (c) $t=0.6$; (d) $t=0.8$ (Nodes: $11 \times 11, d t=0.07$ ) 


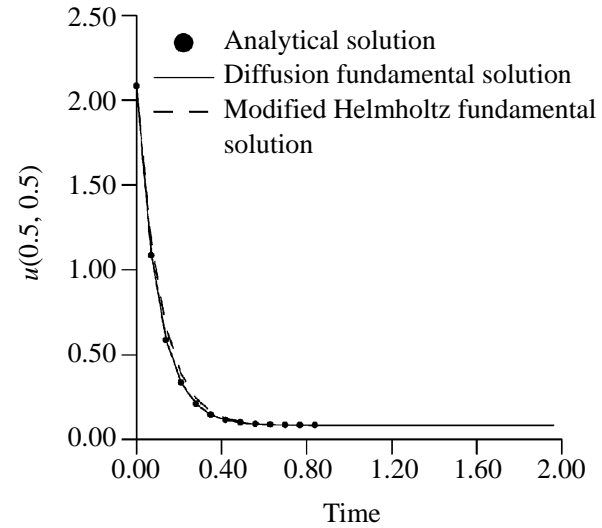

Fig. 5 Comparison of time evolution of $u$ at $x=y=0.5$ (Nodes: $11 \times 11, d t=0.07)$

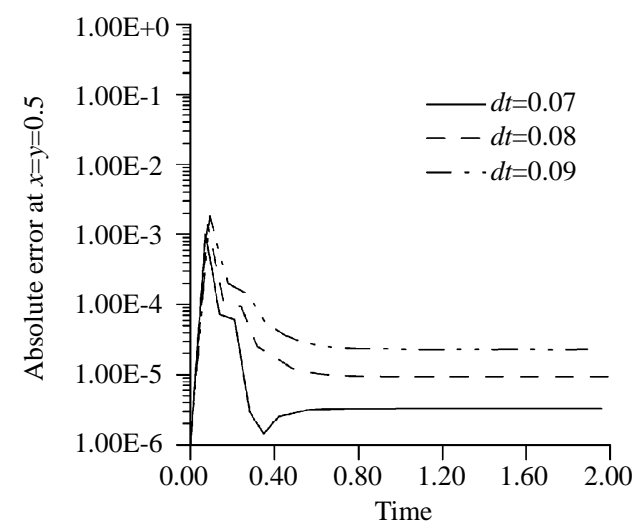

(a) Node $11 \times 11$

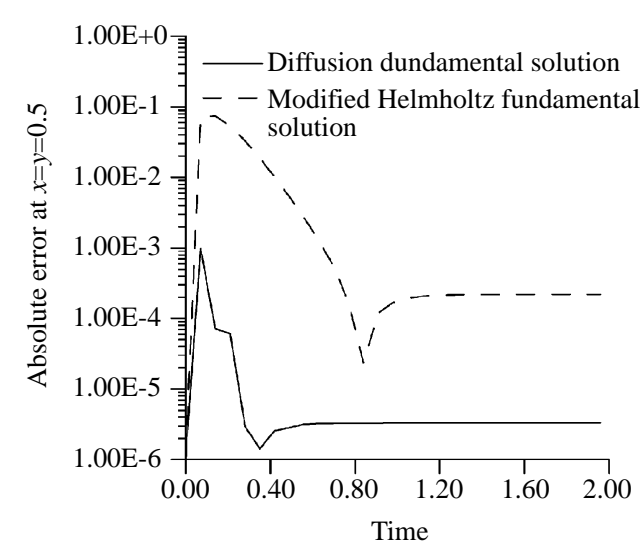

Fig. 6 Comparison of absolute error of $u$ at $x=y=0.5$ (Nodes: $11 \times 11, d t=0.07$ )

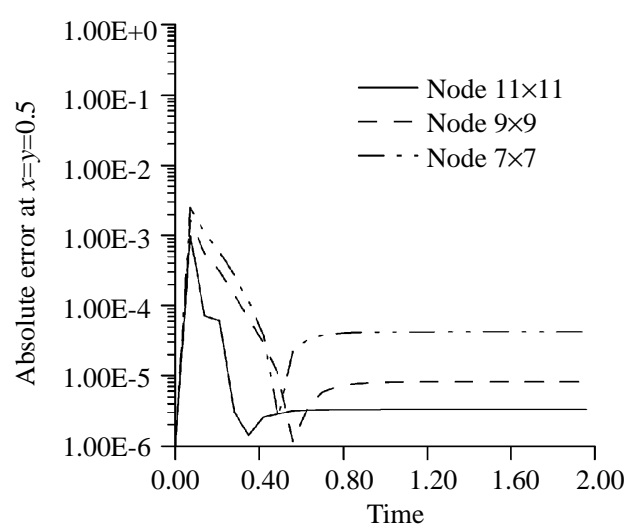

(b) $d t=0.07$

Fig. 7 Time evolution history of absolute error of $u$ at $x=y=0.5$ by the MFS-DRM model based on diffusion fundamental solution

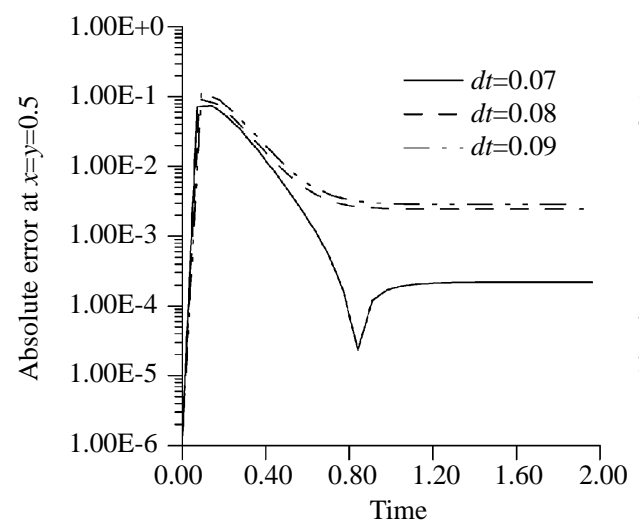

(a) Node $11 \times 11$

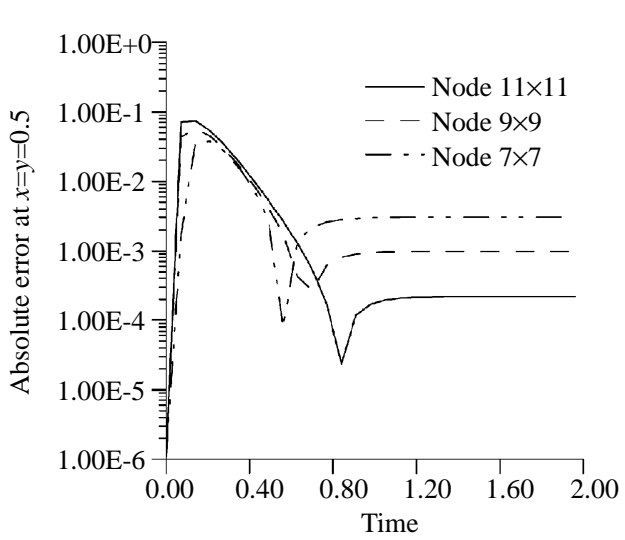

(b) $d t=0.07$

Fig. 8 Time evolution history of absolute error of $u$ at $x=y=0.5$ by the MFS-DRM model based on modified Helmholtz fundamental solution

Helmholtz fundamental solution. In Fig. 6, the MFSDRM model based on the diffusion fundamental solution shows better performance than the MFS-DRM model based on the modified Helmholtz fundamental solution, since the diffusion fundamental solution is a time-dependent function, which is capable of cap- turing the transient process much better (two order accuracy). On the other hand, Fig. 7 and Fig. 8 depict the error histograms for the two methods for different numbers of points and different time increments, in which more points and smaller time increments generally give better results, as expected. 


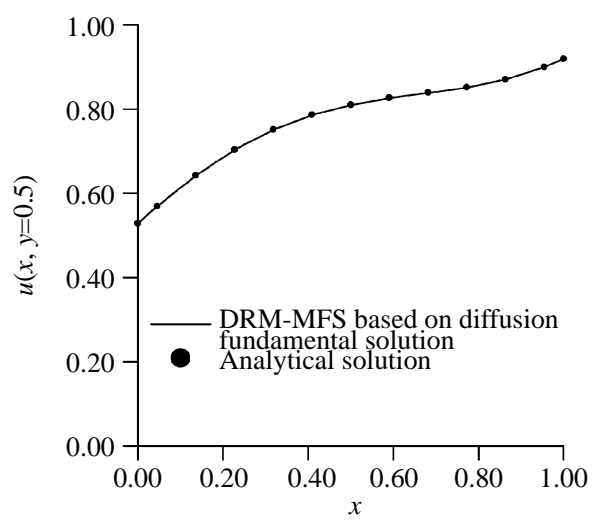

(a) $t=0.2$

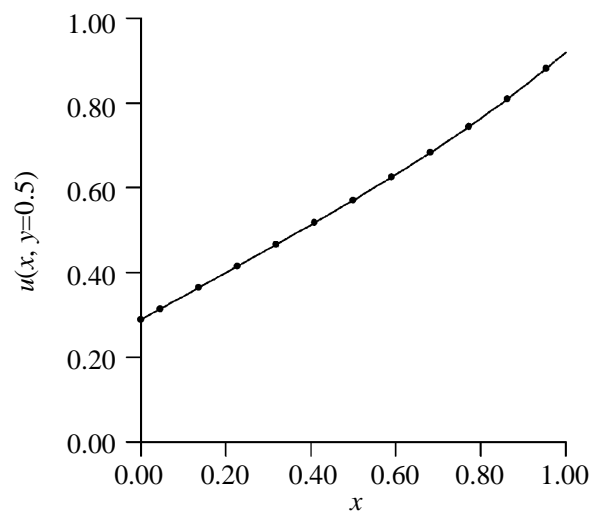

(b) $t=0.4$

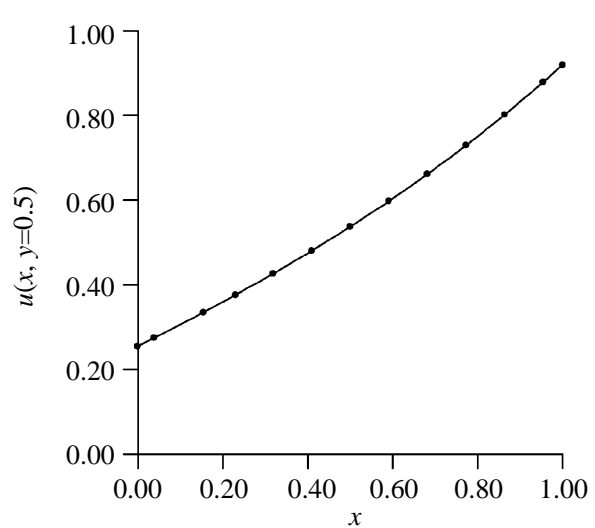

(c) $t=0.6$

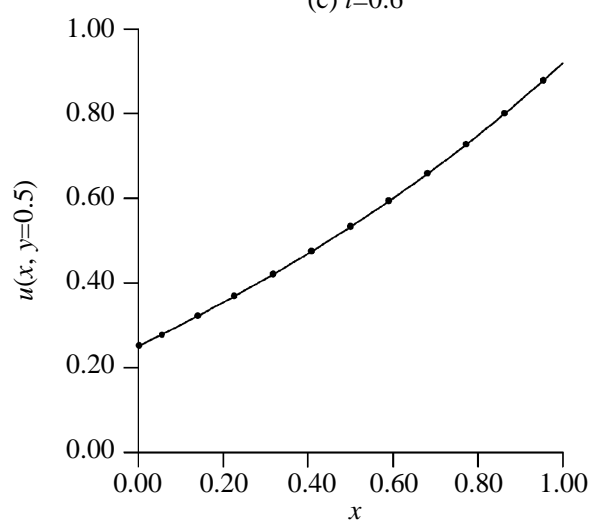

(d) $t=0.8$

Fig. 9 Comparison of $u$-distribution along $y=0.5$ by the MFS-DRM model based on diffusion fundamental solution (a) $t=0.2$; (b) $t=0.4$; (c) $t=0.6$; (d) $t=0.8$ (Nodes: $11 \times 11, d t=0.07$ )

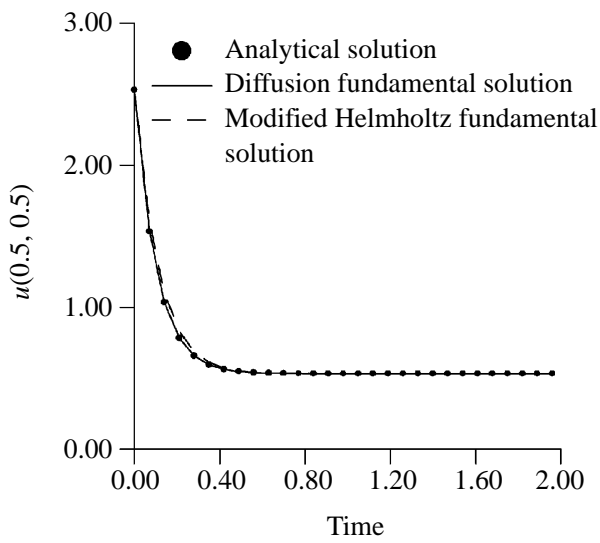

Fig. 10 Comparison of time evolution of $u$ at $x=y=0.5$ (Nodes: $11 \times 11, d t=0.07)$

\section{Example 2:}

G.E.: $\frac{\partial u}{\partial t}=\nabla^{2} u-\frac{y e^{x}-x \sin [y]}{2}$

I.C.: $u(x, y, t=0)=\cos [\pi x]+\cos [\pi y]+\sin [\pi x]$

$$
+\sin [\pi y]+\frac{y e^{x}+x \sin [y]}{2}
$$

$$
B . C .:\left\{\begin{aligned}
u(0, y, t)= & (1+\cos [\pi y]+\sin [\pi y]) e^{-\pi^{2} t}+\frac{y}{2} \\
u(1, y, t)= & (-1+\cos [\pi y]+\sin [\pi y]) e^{-\pi^{2} t} \\
& +\frac{y e+\sin [y]}{2} \\
u(x, 0, t)= & (\cos [\pi x]+1+\sin [\pi x]) e^{-\pi^{2} t} \\
u(x, 1, t)= & (\cos [\pi x]-1+\sin [\pi x]) e^{-\pi^{2} t} \\
& +\frac{e^{x}+x \sin [1]}{2}
\end{aligned}\right.
$$

The analytical solution of the problem is given by

$$
\begin{aligned}
u(x, y, t)= & (\cos [\pi x]+\cos [\pi y]+\sin [\pi x] \\
& +\sin [\pi y]) e^{-\pi^{2} t}+\frac{y e^{x}+x \sin [y]}{2}
\end{aligned}
$$

The numerical results of this example are shown in Figs. 9-13. They are similar to the previous example. The results of the MFS-DRM model based on the diffusion fundamental solution also show very good agreement with the analytical solution and demonstrate better performance than the MFS-DRM model based on the modified Helmholtz fundamental solution.

\section{3D Diffusion Problem}

For the last problem, the proposed numerical 


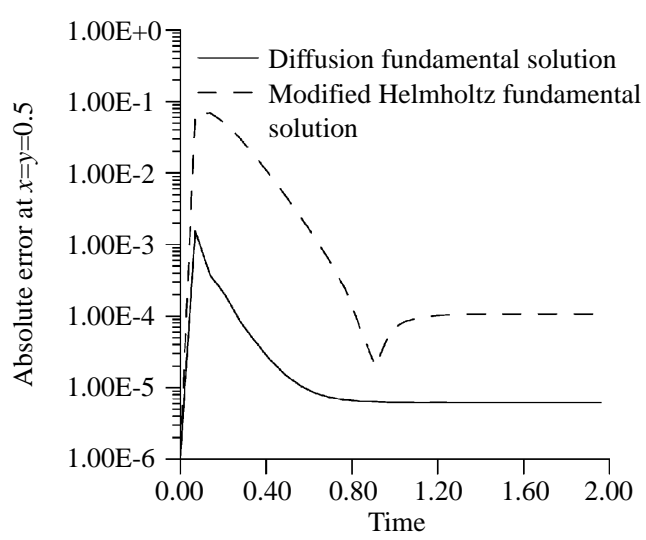

Fig. 11 Comparison of absolute error of $u$ at $x=y=0.5$ (Nodes: $11 \times 11, d t=0.07$ )

method is extended to study $3 \mathrm{D}$ diffusion problem inside a solid cube with dimensions $[0,1] \times[0,1] \times[0,1]$.

\section{Example 3:}

G.E.: $\frac{\partial u}{\partial t}=\nabla^{2} u+\frac{\sin [y]-e^{x}-e^{z}-6 x-6 y-6 z}{10}$

I.C.: $u(x, y, z, t=0)=\sin [\pi x]+\sin [\pi y]+\sin [\pi z]$

$$
+\frac{e^{x}+\sin [y]+e^{z}+x^{3}+y^{3}+z^{3}}{10}
$$

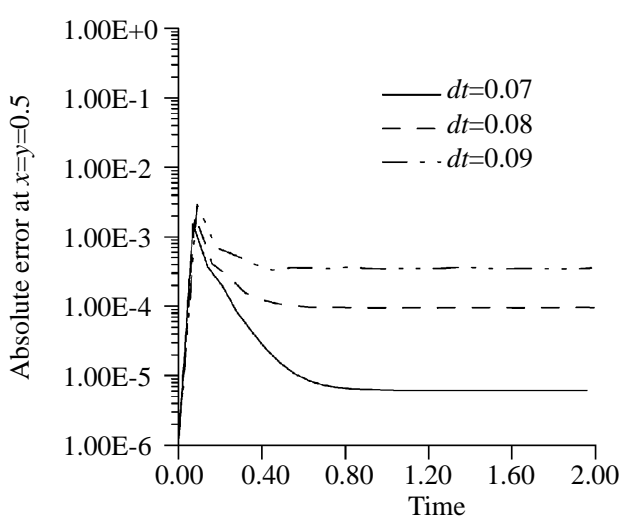

(a) Nodes $11 \times 11$

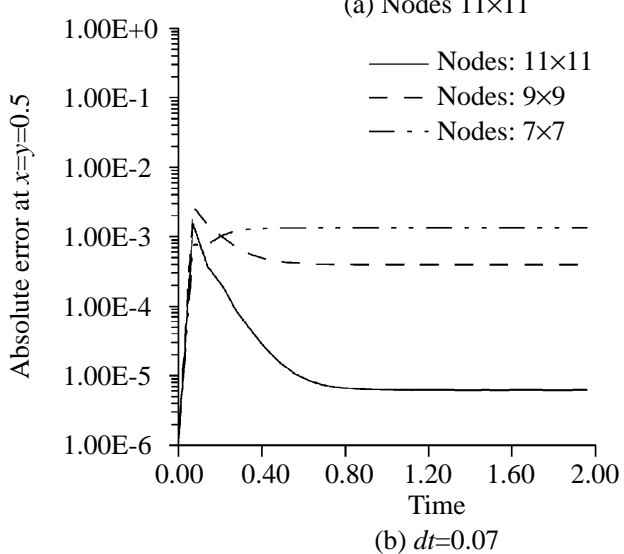

Fig. 12 Time evolution history of absolute error of $u$ at $x=y=0.5$ by the MFS-DRM model based on diffusion fundamental solution

$$
\text { B.C.: }\left\{\begin{array}{l}
u(0, y, z, t)=(\sin [\pi y]+\sin [\pi z]) e^{-\pi^{2} t}+\frac{1+\sin [y]+e^{z}+y^{3}+z^{3}}{10} \\
u(1, y, z, t)=(\sin [\pi y]+\sin [\pi z]) e^{-\pi^{2} t}+\frac{e+\sin [y]+e^{z}+1+y^{3}+z^{3}}{10} \\
u(x, 0, z, t)=(\sin [\pi x]+\sin [\pi z]) e^{-\pi^{2} t}+\frac{e^{x}+e^{z}+x^{3}+z^{3}}{10} \\
u(x, 1, z, t)=(\sin [\pi x]+\sin [\pi z]) e^{-\pi^{2} t}+\frac{e^{x}+\sin [1]+e^{z}+x^{3}+1+z^{3}}{10} \\
u(x, y, 0, t)=(\sin [\pi x]+\sin [\pi y]) e^{-\pi^{2} t}+\frac{e^{x}+\sin [y]+1+x^{3}+y^{3}}{10} \\
u(x, y, 1, t)=(\sin [\pi x]+\sin [\pi y]) e^{-\pi^{2} t}+\frac{e^{x}+\sin [y]+e+x^{3}+y^{3}+1}{10}
\end{array}\right.
$$

The analytical solution of the problem is given by

$$
\begin{aligned}
u(x, y, z, t)= & (\sin [\pi x]+\sin [\pi y]+\sin [\pi z]) e^{-\pi^{2} t} \\
& +\frac{e^{x}+\sin [y]+e^{z}+x^{3}+y^{3}+z^{3}}{10}
\end{aligned}
$$

Figure 14 shows the time evolution history at (0.5. $0.5,0.5)$ of the solution for the MFS-DRM model based on the diffusion fundamental solution as well as the MFS-DRM model based on the modified Helmholtz fundamental solution. On the other hand, Fig. 15 shows the time evolution history of the absolute error for the two methods, in which the MFSDRM model based on the diffusion fundamental solution, which has better performance. Finally, Fig. 16 and Fig. 17 depict the error histograms for the two methods for different numbers of points and 


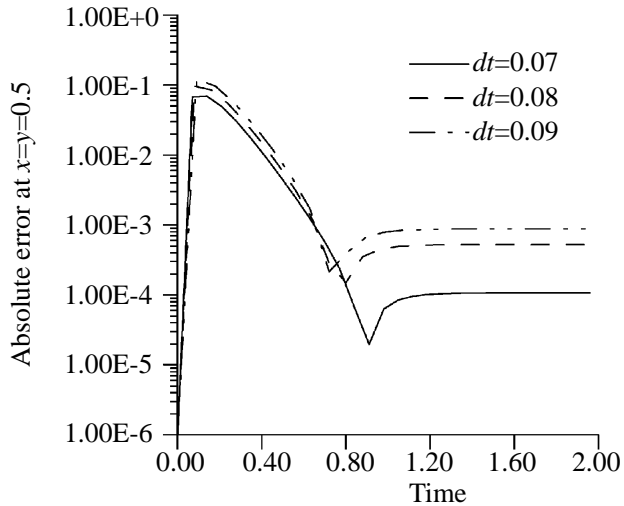

(a) Nodes $11 \times 11$

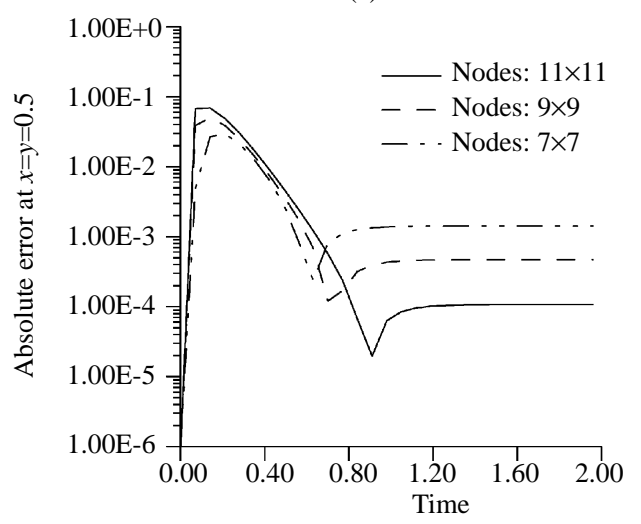

(b) $d t=0.07$

Fig. 13 Time evolution history of absolute error of $u$ at $x=y=0.5$ by the MFS-DRM model based on modified Helmholtz fundamental solution

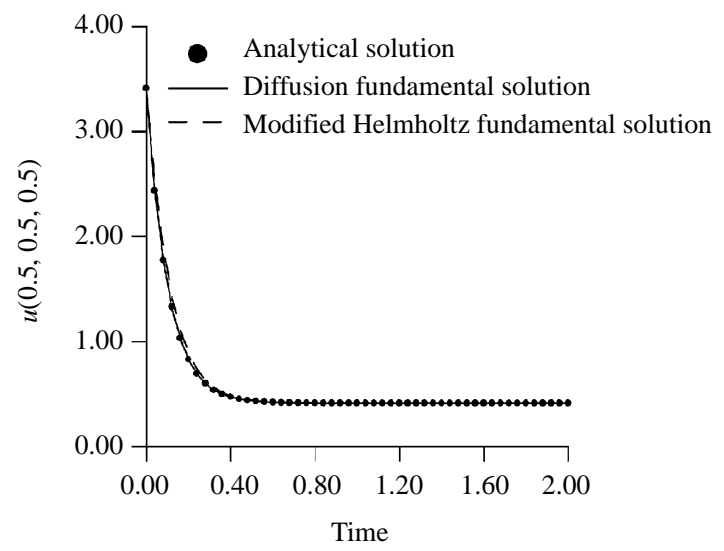

Fig. 14 Comparison of time evolution of $u$ at $x=y=z=0.5$ (Nodes: $7 \times 7 \times 7, d t=0.04)$

different time increments, in which more points and smaller time increments in general will give better results, as before.

\section{CONCLUSIONS}

Transient diffusion problems with time-independent source terms in multi-dimensions are solved

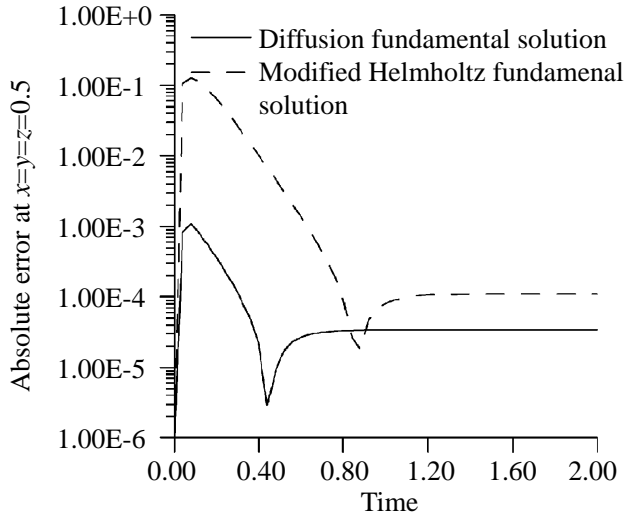

Fig. 15 Comparison of time evolution of absolute error of $u$ at $x=y=z=0.5$ (Nodes: $7 \times 7 \times 7, d t=0.04)$

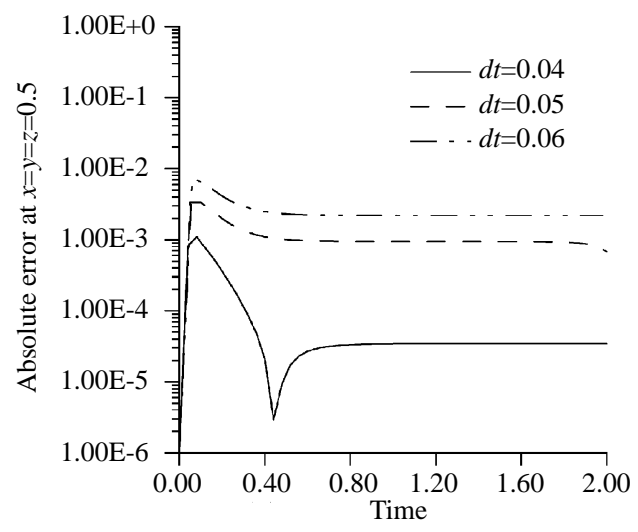

(a) Nodes $7 \times 7 \times 7$

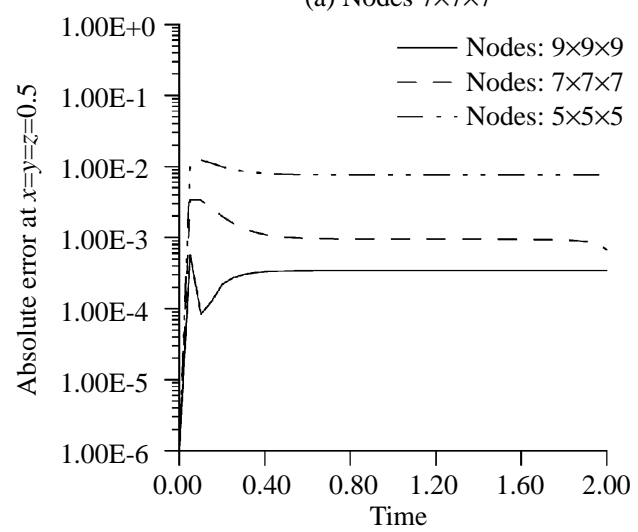

(b) $d t=0.05$

Fig. 16 Time evolution history of absolute error of $u$ at $x=y=z=0.5$ by the MFS-DRM model based on diffusion fundamental solution

using the MFS-DRM model based on the direct usage of the diffusion fundamental solution. The MFS is adopted to obtain the homogeneous solution and the DRM is utilized to solve the nonhomogeneous source term. The generally adopted Laplace transform and finite difference scheme for the time derivative term are not required in the proposed numerical solution procedure. The independent time 


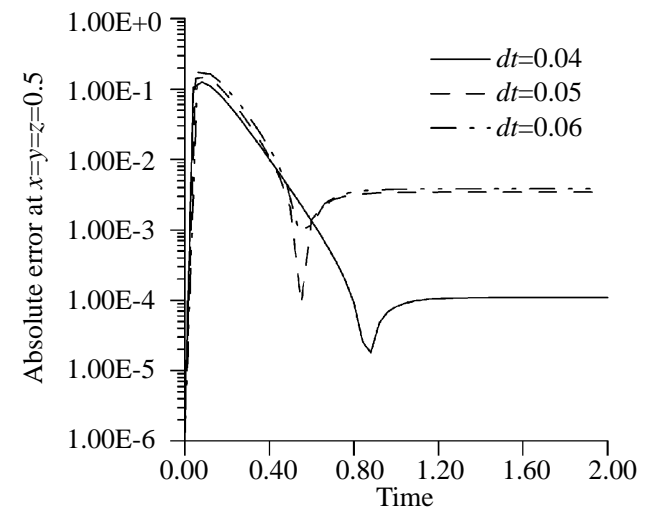

(a) Nodes $7 \times 7 \times 7$

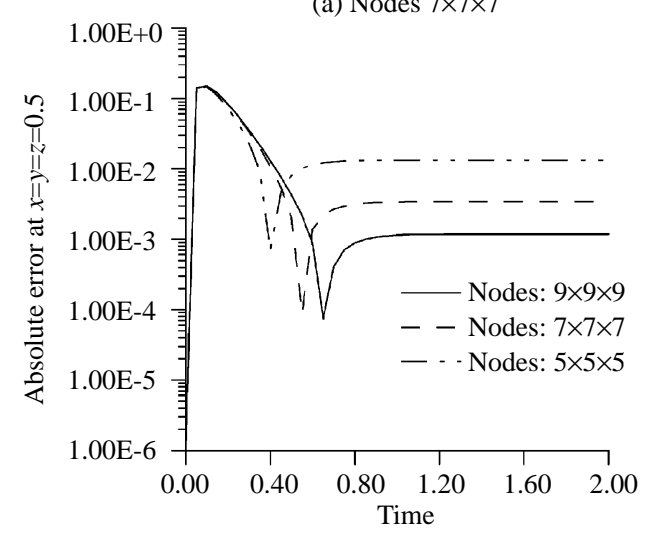

(b) $d t=0.05$

Fig. 17 Time evolution history of absolute error of $u$ at $x=y=z=0.5$ by the DRM-MFS based on modified Helmholtz fundamental solution

variable was treated as one of the solution domains in the time-dependent fundamental solutions and this space-time unification makes it possible to make use of the MFS to obtain the numerical solutions of the homogeneous diffusion equation without transformation or difference discretization for the time domain. By properly locating the source points and the field points at every time level, the solutions were advanced in time until the system reached steady state conditions. The numerical procedure developed in the present work was validated by comparing its results with the results obtained by analytical solutions as well as the traditional MFS-DRM model based on the modified Helmholtz fundamental solution for 2D and 3D diffusion problems under the Dirichlet boundary conditions. The excellent agreement with the analytical results and better performance than the traditional method indicate the effectiveness of the present method to solve diffusion equations with time-independent source terms without requiring any time transformation or time discretization. Besides, the unification of time and space variables makes the removal of singularities with respect to both space and time except $t-\tau=0$ possible.

\section{ACKNOWLEDGMENTS}

The National Science Council of Taiwan is gratefully acknowledged for providing financial support of NSC 92-2611-E-002-007 to carry out the present work.

\section{REFERENCES}

Atluri, S. N., and Zhu, T., 1998, “A New Meshless Local Petrov-Galerkin (MLPG) Approach in Computational Mechanics," Computational Mechanics, Vol. 22, No. 2, pp. 117-127.

Balakrishnan, K., and Ramachandran, P. A., 2000, "The Method of Fundamental Solutions for Linear Diffusion-Reaction Equations," Mathematical and Computer Modeling, Vol. 31, Nos. 2-3, pp. 221-237.

Berger, J. R., and Karageorghis, A., 1999, "The Method of Fundamental Solutions for Heat Conduction in Layered Materials," International Journal for Numerical Methods in Engineering, Vol. 45, No. 11, pp. 1681-1694.

Bialecki, R. A., Jurgas, P., and Kuhn, G., 2002, “Dual Reciprocity BEM without Matrix Inversion for Transient Heat Conduction," Engineering Analysis with Boundary Elements, Vol. 26, No. 3, pp. 227-236.

Bulgakov, V., Sarler, B., and Kuhn, G., 1998, "Iterative Solution of Systems of Equations in the Dual Reciprocity Boundary Element Method for the Diffusion Equation," International Journal for Numerical Methods in Engineering, Vol. 43, No. 4, pp. 713-732.

Chawla, M. M., and Al-Zanaidi, M. A., 1999, “An Extended Trapezoidal Formula for the Diffusion Equation," Computers and Mathematics with Applications, Vol. 38, pp. 51-59.

Chen, C. S., Golberg, M. A., and Hon, Y. C., 1998a, "The Method of Fundamental Solutions and Quasi-Monte-Carlo Method for Diffusion Equations," International Journal for Numerical Methods in Engineering, Vol. 43, No. 8, pp. 14211435.

Chen, C. S., Golberg, M. A., and Rashed, Y. F., 1998b, "A Mesh Free Method for Linear Diffusion Equations," Numerical Heat Transfer Part $B$, Vol. 33, pp. 469-486.

Chen, J. T., Hong, H.-K., Yeh, C. S., and Chyuan, S. W.,1996, "Integral Representations and Regularizations for a Divergent Series Solution of a Beam Subjected to Support Motions," Earthquake Engineering and Structural Dynamics, Vol. 25, pp. 909-925.

Golberg, M. A., 1995, "The Method of Fundamental Solutions for Poisson's Equation," Engineering 
Analysis with Boundary Elements, Vol. 16, No. 3, pp. 205-213.

Golberg, M. A., and Chen, C. S., 1998, "The Method of Fundamental Solutions for Potential, Helmholtz and Diffusion Problems," Boundary Integral Methods, Computational Mechanics Publications, Boston, U.S.A., pp. 103-176.

Hobson, J. M., Wood, N., and Mason, P. J., 1996, “A New Finite-Difference Diffusion Scheme," Journal of Computational Physics, Vol. 125, No. 1, pp. 16-25.

Jones, W. P., and Menzies, K. R., 2000, "Analysis of the Cell-Centered Finite Volume Method for the Diffusion Equation," Journal of Computational Physics, Vol. 165, No. 1, pp. 45-68.

Kansa, E. J., 1990a, "Multiquadrics - a Scattered Data Approximation Scheme with Applications to Computational Fluid Dynamics-I, Surface Approximations and Partial Derivative Estimates," Computers and Mathematics with Applications, Vol. 19, Nos. 8-9, pp. 127-145.

Kansa, E. J., 1990b, "Multiquadrics - a Scattered Data Approximation Scheme with Applications to Computational Fluid Dynamics II, Solutions to Parabolic, Hyperbolic and Elliptic Partial Differential Equations," Computers and Mathematics with Applications, Vol. 19, Nos. 8-9, pp. 147-161.

Kim, H. G., and Atluri, S. N., 2000, “Arbitrary Placement of Secondary Nodes, and Error Control, in the Meshless Local Petrov-Galerkin (MLPG) Method," Computer Modeling in Engineering and Sciences, Vol. 1, No. 3, pp. 11-32.

Lin, H., and Atluri, S. N., 2000, "Meshless Local Petrov-Galerkin (MLPG) Method for ConvectionDiffusion Problems," Computer Modeling in Engineering and Sciences, Vol. 1, No. 2, pp. 45-60.

Lin, H., and Atluri, S. N., 2001, "The Meshless Local Petrov-Galerkin (MLPG) Method for Solving Incompressible Navier-Stokes Equations," Computer Modeling in Engineering and Sciences, Vol. 2, No. 2, pp. 117-142.

Madych, W., 1992, "Miscellaneous Error Bounds for Multiquadric and Related Interpolants," Computers and Mathematics with Applications, Vol. 24, pp. 121-138.

Nardini, D., and Brebbia, C. A., 1982, “A New Approach to Free Vibration Analysis Using Boundary Elements," Boundary Element Methods in Engineering, Springer-Verlag, Berlin, German.

Oden, J. T., Babuska, I., and Baumann, C. E., 1998, "A Discontinuous hp Finite Element Method for Diffusion Problems," Journal of Computational Physics, Vol. 146, No. 2, pp. 491-519.

Poullikkas, A., Karageorghis, A., and Georgiou, G., 1998a, "Methods of Fundamental Solutions for Harmonic and Biharmonic Boundary Value
Problems," Computational Mechanics, Vol. 21, Nos. 4-5, pp. 416-423.

Poullikkas, A., Karageorghis, A., and Georgiou, G., 1998b, "The Method of Fundamental Solutions for Inhomogeneous Elliptic Problems," Сотриtational Mechanics, Vol. 22, No. 1, pp. 100-107.

Ramachandran, P. A., 2002, "Method of Fundamental Solutions: Singular Value Decomposition Analysis," Communications in Numerical Methods in Engineering, Vol. 18, pp. 789-801.

Smyrlis, Y. S., Karageorghis, A., and Georgiou, G., 2001, "Some Aspects of the One-Dimensional Version of the Method of Fundamental Solutions," Computers and Mathematics with Applications, Vol. 41, pp. 647-657.

Sutradhar, A., Paulino, G. H., and Gray, L. J., 2002, "Transient Heat Conduction in Homogeneous and Non-Homogeneous Materials by the Laplace Transform Galerkin Boundary Element Method," Engineering Analysis with Boundary Elements, Vol. 26, No. 3, pp. 119-132.

Teixeira, J., 1999, "Stable Schemes for Partial Differential Equations: the One-Dimensional Diffusion Equation," Journal of Computational Physics, Vol. 153, No. 2, pp. 403-417.

Wordelman, C. J., Atluri, S. N., and Ravaioli, U., 2000, "A Meshless Method for the Numerical Solution of the 2D and 3D Semiconductor Poisson Equation," Computer Modeling in Engineering and Sciences, Vol. 1, No. 1, pp. 121-126.

Zerroukat, M., 1999, "A Boundary Element Scheme for Diffusion Problems Using Compactly Supported Radial Basis Functions," Engineering Analysis with Boundary Elements, Vol. 23, No. 3, pp. 201-209.

Zhu, S. P., 1998, "Solving Transient Diffusion Problems: Time-Dependent Fundamental Solution Approaches Versus LTDRM Approaches," Engineering Analysis with Boundary Elements, Vol. 21, No. 1, pp. 87-90.

Zhu, S. P., Liu, H. W., and Lu, X. P., 1998, "A Combination of LTDRM and ATPS in Solving Diffusion Problems," Engineering Analysis with Boundary Elements, Vol. 21, No. 3, pp. 285-289.

Zhu, T., Zhang, J., and Atluri, S. N., 1998, "A Meshless Local Boundary Integral Equation (LBIE) Method for Solving Nonlinear Problems," Computational Mechanics, Vol. 22, No. 2, pp. 174-176.

Manuscript Received: Dec. 12, 2003 Revision Received: Apr. 25, 2004 and Accepted: May 07, 2004 APPENDIX

Here, we revisit the MFS-DRM model based on 
the modified Helmholtz fundamental solution (Chen et al., 1998b; Golberg and Chen, 1998). First of all, we apply the finite difference in time to the diffusion Eq. (1). That is

$$
\left.\frac{\partial \Phi(\boldsymbol{x}, t)}{\partial t}\right|_{t=n \Delta t}=\frac{\Phi^{(n+1)}(\boldsymbol{x})-\Phi^{(n)}(\boldsymbol{x})}{\Delta t}
$$

where $\Delta t$ is an a priori constant of the time step, and $\Phi^{(n)}(\boldsymbol{x})$ is defined as $\Phi(\boldsymbol{x}, n \Delta t)$. Applying the definition (A1) to the diffusion Eq. (1), we are capable of obtaining

$$
\begin{array}{r}
\frac{\Phi^{(n+1)}(\boldsymbol{x})-\Phi^{(n)}(\boldsymbol{x})}{\Delta t}=k \nabla^{2} \Phi^{(n+1)}(\boldsymbol{x})+A(\boldsymbol{x}) \text { in } \Omega \\
\text { (A2) } \\
\text { or } \nabla^{2} \Phi^{(n+1)}(\boldsymbol{x})-\lambda^{2} \Phi^{(n+1)}(\boldsymbol{x})=-\lambda^{2} \Phi^{(n)}(\boldsymbol{x})-\frac{A(\boldsymbol{x})}{k} \text { in } \Omega
\end{array}
$$

where $\lambda^{2}=\frac{1}{\Delta t k}$. Combining with the boundary condition (3) and the initial condition (2), this will result in

$$
\begin{aligned}
& \Phi^{(0)}(\boldsymbol{x})=B(\boldsymbol{x}) \text { in } \Omega \\
& \Phi^{(n+1)}(\boldsymbol{x})=C^{(n+1)}(\boldsymbol{x}) \text { on } \Gamma^{1} \\
& \frac{\partial \Phi^{(n+1)}}{\partial n}(\boldsymbol{x})=D^{(n+1)}(\boldsymbol{x}) \text { on } \Gamma^{2}
\end{aligned}
$$

The Eq. (A3) and Eq. (A4) can be viewed as a series of modified Helmholtz equations for the unknown series of functions, $\Phi^{(1)}(x), \Phi^{(2)}(x), \Phi^{(3)}(x)$, with known initial function $\Phi^{(0)}(\boldsymbol{x})=B(\boldsymbol{x})$.

In order to apply the MFS-DRM model based on the modified Helmholtz fundamental solution to solve the equation, we first decompose the unknown function to

$$
\Phi^{(n+1)}(\boldsymbol{x})=\Phi_{h}^{(n+1)}(\boldsymbol{x})+\Phi_{p}^{(n+1)}(\boldsymbol{x})
$$

where the particular solution, $\Phi_{p}^{(n+1)}(\boldsymbol{x})$, satisfies

$$
\begin{gathered}
\nabla^{2} \Phi_{p}^{(n+1)}(\boldsymbol{x})-\lambda^{2} \Phi_{p}^{(n+1)}(\boldsymbol{x})=-\lambda^{2} \Phi^{(n)}(\boldsymbol{x})-\frac{A(\boldsymbol{x})}{k} \\
\text { in } \Omega
\end{gathered}
$$

and the homogeneous solution, $\Phi_{h}^{(n+1)}(\boldsymbol{x})$, satisfies

$$
\nabla^{2} \Phi_{h}^{(n+1)}(\boldsymbol{x})-\lambda^{2} \Phi_{h}^{(n+1)}(\boldsymbol{x})=0 \text { in } \Omega
$$

$$
\begin{aligned}
& \Phi_{h}^{(n+1)}(\boldsymbol{x})=C^{(n+1)}(\boldsymbol{x})-\Phi_{p}^{(n+1)}(\boldsymbol{x}) \text { in } \Gamma^{1} \\
& \frac{\partial \Phi_{h}^{(n+1)}}{\partial n}(\boldsymbol{x})=D^{(n+1)}(\boldsymbol{x})-\frac{\partial \Phi_{p}^{(n+1)}}{\partial n}(\boldsymbol{x}) \text { in } \Gamma^{2}
\end{aligned}
$$

The particular solution corresponding to Eq. (A6) can be approximated by the DRM for the source term $-\lambda^{2} \Phi^{(n)}(\boldsymbol{x})-\frac{A(\boldsymbol{x})}{k}$

$$
\begin{aligned}
& -\lambda^{2} \Phi^{(n)}(\boldsymbol{x})-\frac{A(\boldsymbol{x})}{k} \\
& = \begin{cases}\sum_{j=1}^{N} a_{j} r_{i j}^{2} \operatorname{Ln}\left[r_{i j}\right]+b_{1} x+b_{2} y+b_{3} \text { for } 2 D \\
\sum_{j=1}^{N} a_{j} r_{i j}+b_{1} x+b_{2} y+b_{3} z+b_{4} \text { for } 3 D\end{cases}
\end{aligned}
$$

in which the details are the same as the DRM for the diffusion equation posted before. Therefore, the particular solution $\Phi_{p}^{(n+1)}(\boldsymbol{x})$ can be determined (Golberg and Chen, 1998):

$$
\begin{aligned}
& \Phi_{p}^{(n+1)}(\boldsymbol{x}) \\
& = \begin{cases}\sum_{j=1}^{N} a_{j}\left\{\frac{-4}{\lambda^{4}}\left(K_{0}\left(\lambda r_{i j}\right)+\operatorname{Ln}\left[r_{i j}\right]\right)\right. & \\
\left.-\frac{r_{i j}^{2} L n\left[r_{i j}\right]}{\lambda^{2}}\right\}-\frac{4}{\lambda^{4}}-\frac{b_{1} x}{\lambda^{2}}-\frac{b_{2} y}{\lambda^{2}}-\frac{b_{3}}{\lambda^{2}} & \text { for } 2 D \\
\sum_{j=1}^{N} a_{j}\left\{\frac{2}{\lambda^{4} r_{i j}}\left(e^{-\lambda r_{i j}}-1\right)-\frac{r_{i j}}{\lambda^{2}}\right\} & \text { for } 3 D \\
-\frac{b_{1} x}{\lambda^{2}}-\frac{b_{2} y}{\lambda^{2}}-\frac{b_{3} z}{\lambda^{2}}-\frac{b_{4}}{\lambda^{2}} & \end{cases}
\end{aligned}
$$

where $K_{0}(\bullet)$ is the zero order modified Bessel function of the second kind.

With the substitution of the Eq. (A9) into the governing equation of the homogeneous Eq. (A7), the result will be a well-posed homogeneous modified Helmholtz equation, and hence it is capable of being solved. Since the modified Helmholtz fundamental solution satisfies the modified Helmholtz equation, we may assume the homogeneous solution is a linear combination of the fundamental solution of the modified Helmholtz operator, i.e.:

$$
\Phi_{h}^{(n+1)}(\boldsymbol{x})=\sum_{i=1}^{N} c_{i} g\left(\left|\boldsymbol{x}-\xi_{i}\right|\right)
$$

where 


$$
g(r)= \begin{cases}\frac{1}{2 \pi} K_{0}[\lambda r] & \text { for } 2 D \\ \frac{e^{-\lambda r}}{4 \pi r} & \text { for } 3 D\end{cases}
$$

is the fundamental solution of the modified Helmholtz operator (Chen et al., 1998b; Golberg and Chen, 1998), $\boldsymbol{x}$ represents the location of the field points, $\xi_{i}$ gives the location of the source points, $r=\left|x-\xi_{i}\right|$ is the distance, and $N$ is the number of source points and field points. The source points are typically distributed away from the boundary field points to avoid the singularity as shown in Fig. 3. By collocating these field points and using Eq. (A7), a linear matrix system can be formed as follows

$$
\left\lfloor A_{i j}\right\rfloor\left\{c_{j}\right\}=\left\{d_{i}\right\}
$$

$$
\text { where } A_{i j}= \begin{cases}\frac{1}{2 \pi} K_{0}\left[\lambda\left|\boldsymbol{x}_{i}-\xi_{j}\right|\right] & \text { for } 2 D \\ \frac{e^{-\lambda\left|x_{i}-\xi_{j}\right|}}{4 \pi\left|x_{i}-\xi_{j}\right|} & \text { for } 3 D\end{cases}
$$

The vector $\left\{d_{i}\right\}$ stems from the boundary conditions. After inverting the matrix system, the coefficients $\left\{c_{j}\right\}$ can be obtained, and then the homogenous solution at $t=(n+1) \Delta t$ can be acquired.

After the homogenous solution and the particular solution are solved, the solution of the original diffusion equation at $t=(n+1) \Delta t$ can be obtained by the superposition principle of Eq. (A5). Therefore, the procedure can be repeated until either the terminal time or a steady state solution is achieved. 